\title{
artigo
}

Brito, F.C.R.; Morira, M.R.; Ferreira da Silva, M.F.; Andrade, V.O.A.; Lima, W.O.O.

Perfil antropometrico e o risco de doenças cardiovasculares entre manipuladores de alimentos

\section{Perfil antropometrico e o risco de doenças cardiovasculares entre manipuladores de alimentos}

\author{
Anthropometric profile and the risk of cardiovascular diseases among food handlers \\ Perfil antropométrico y riesgo de enfermedades cardiovasculares en los manipuladores de alimentos
}

\begin{abstract}
RESUMO
Objetivo: Caracterizar o perfil antropométrico de manipuladores de alimentos. Métodos: Foram aferidos: o peso, altura e a circunferência da cintura (CC) e relacionadas com os parâmetros determinados pela Organização Mundial de Saúde (OMS) para o risco de doenças cardiovasculares (RC). Foi realizado um questionário contendo perguntas sobre o nível de escolaridade, a prática de exercícios físicos e o diagnóstico de doenças crônicas. Resultados: 0 estudo contou com a participação dos 28 colaboradores da unidade. 0 cálculo do Índice de Massa corpórea (IMC) demonstrou que 12 colaboradores (42,85\%) apresentaram classificação de Obesidade Grau. O RC foi alto para 17 colaboradores $(60,71 \%$ ). Conclusão: Os manipuladores de alimentos pesquisados apresentaram graves fatores de risco para o desenvolvimento de doenças cardiovasculares de acordo com os critérios determinados pela OMS. Esperamos que trabalhos como este auxiliem na caracterização do perfil nutricional dos trabalhadores e na elaboração de propostas que melhorem sua qualidade de vida.
\end{abstract}

DESCRITORES: Saúde do trabalhador; Doenças Crônicas Não Transmissiveis; Alimentação Coletiva.

\section{ABSTRACT}

Objective: To characterize the anthropometric profile of food handlers. Methods: Weight, height and waist circumference (WC) were measured and related to the parameters determined by the World Health Organization (WHO) for the risk of cardiovascular diseases (CR). A questionnaire was conducted containing questions about education level, physical exercise and the diagnosis of chronic diseases. Results: The study involved the participation of 28 employees at the unit. The calculation of the Body Mass Index (BMI) showed that 12 employees (42.85\%) had a Classification of Obesity Degree. The RC was high for 17 employees (60.71\%). Conclusion: The food handlers surveyed presented serious risk factors for the development of cardiovascular diseases according to the criteria determined by the WHO. We hope that works like this help to characterize the nutritional profile of workers and to develop proposals that improve their quality of life.

DESCRIPTORS: Worker's health; Chronic Noncommunicable Diseases; Collective Food.

\section{RESUMEN}

Objetivo: caracterizar el perfil antropométrico de los manipuladores de alimentos. Métodos: Se midieron el peso, la talla y la circunferencia de la cintura (CC) y se relacionaron con los parámetros determinados por la Organización Mundial de la Salud (OMS) para el riesgo de enfermedades cardiovasculares (RC). Se realizó un cuestionario con preguntas sobre nivel educativo, ejercicio físico y diagnóstico de enfermedades crónicas. Resultados: El estudio contó con la participación de 28 empleados de la unidad. El cálculo del Îndice de Masa Corporal (IMC) mostró que 12 empleados (42,85\%) tenían un Grado de Clasificación de Obesidad. El RC fue alto para 17 empleados $(60,71 \%)$. Conclusión: Los manipuladores de alimentos encuestados presentaron graves factores de riesgo para el desarrollo de enfermedades cardiovasculares según los criterios determinados por la OMS. Esperamos que trabajos como este ayuden a caracterizar el perfil nutricional de los trabajadores y a desarrollar propuestas que mejoren su calidad de vida.

DESCRIPTORES: Salud del trabajador; Enfermedades crónicas no transmisibles; Alimentación colectiva.

RECEBIDO EM: 01/02/2021 APROVADO EM: 16/02/2021

\section{Fernando César Rodrigues Brito}

Nutricionista. Doutor em Biotecnologia e Saúde. Docente do curso de Nutrição da Universidade Federal do Rio Grande do Norte - Campus FACISA.

ORCID: 0000-0001-8181-664X 


\section{Marta da Rocha Moreira}

Nutricionista. Mestre em Ciências Fisiológicas. Docente dos cursos de Nutrição do Centro Universitário Estácio do Ceará e da Universidade de Fortaleza

ORCID: 0000-0001-8181-664x

\section{Maria Flávia Ferreira da Silva}

Discente do curso de Nutrição da Universidade de Fortaleza.

ORCID: 0000-0003-0300-4915

\section{Mariana de Lima Matos}

Discente do curso de Nutrição da Universidade de Fortaleza.

ORCID: 0000-0003-2920-4499

\section{Vitória de Oliveira Almeida Andrade}

Nutricionista do Hospital de Saúde Mental Professor Frota Pinto. Fortaleza- CE ORCID: 0000-0003-4580-788X

\section{Wanessa Oliveira de Lima e Lima}

Discente do curso de Nutrição do Centro Universitário Maurício de Nassau

ORCID: 0000-0002-4081-4733

\section{INTRODUÇÃO}

A s unidades de Alimentação e $\mathrm{Nu}-$ trição (UAN) são caracterizadas pela elaboração de refeições para comunidades sadias ou enfermas, de acordo com o objetivo do estabelecimento. Manipuladores de alimentos é a denominação dada aos trabalhadores que manipulam e transformam a matéria-prima, desde o seu recebimento até a distribuição da refeição. ${ }^{1,2}$

Vários estudos relatam que grande parte desses manipuladores estão sujeitos a uma carga horária de trabalho excessiva em diferentes turnos de trabalho. As condições de trabalho extenuantes, juntamente com pouco cuidado com a saúde, submetem esses trabalhadores a diversos riscos, dentre eles, os advindos de uma alimentação de má-qualidade. ${ }^{3}$

$\mathrm{O}$ excesso de peso, tabagismo, sedentarismo; assim como os baixos níveis de escolaridade e de renda são alguns dos fatores que interferem na qualidade de vida dos trabalhadores e estão relacionados com o risco de doenças crônicas não transmissíveis como a diabetes, obesidade e a hipertensão. ${ }^{4}$

A Organização Mundial de Saúde (OMS) propõe parâmetros antropométricos que relacionam o risco de acometimento desses agravos na população. Esses pa-
As unidades de

Alimentação e

Nutrição (UAN)

são caracterizadas

pela elaboração

de refeições para

comunidades

sadias ou enfermas,

de acordo com

o objetivo do

\section{estabelecimento.}

râmetros possuem baixo custo, são de fácil aferição e devem estar relacionados com o cuidado e a saúde dos trabalhadores 5,7
Este estudo tem como objetivo realizar a caracterização do perfil antropométrico dos colaboradores de uma Unidade de Alimentação e Nutrição hospitalar de Fortaleza-CE e assim, estimar o risco de doenças cardiovasculares de acordo com as recomendações da OMS.

\section{MÉTODOS}

Trata-se de uma pesquisa transversal, quantitativa e descritiva, realizada no Centro de Nutrição do Hospital de Saúde Mental Professor Frota Pinto (HSM), situado na cidade de Fortaleza-Ce no período de novembro a dezembro de 2020 . A unidade possuía um total de 28 colaboradores, todos na faixa etária adulta (entre 18 e 59 anos de idade). Não havia nenhuma colaborada gestante no período da pesquisa.

O perfil nutricional foi analisado por meio das variáveis: peso, altura, circunferência do quadril e cintura (CC), Índice Cintura/Quadril (ICQ) e Índice de Massa Corporal (IMC). O peso foi coletado com balança digital Tanitå, capacidade de 150 $\mathrm{kg}$ e precisão de $100 \mathrm{~g}$.

A estatura foi medida com estadiômetro Alturaexata, precisão de $0,1 \mathrm{~cm}$; e as medidas de circunferência obtidas com fita métrica inelástica Sanny". Todas as 
medidas foram coletadas em duplicata, seguindo as normas técnicas descritas na literatura. ${ }^{6}$ As aferições foram realizadas por um único avaliador/pesquisador, sempre com os mesmos equipamentos, estando esses devidamente calibrados.

O questionário estruturado contendo informações sobre idade, função exercida, diagnóstico de doenças crônicas não transmissíveis (diabetes tipo 2, dislipidemia ou hipertensão), prática de atividade física e nível de escolaridade foi preenchido pelo pesquisador após entrevista com os participantes da pesquisa. Esta entrevista, ocorreu durante os intervalos do turno de trabalho em local separado da unidade produtiva, de forma individualizada.

A OMS sugere a avaliação do perfil antropométrico como medida eficaz para avaliar o risco de doenças crônicas não transmissíveis ${ }^{5,7}$, como já dito anteriormente. Os valores de referências indicativos são separados por sexo de acordo com o quadro abaixo

Após a coleta, os dados obtidos foram compilados e tabulados em e submetidos posteriormente ao cálculo de porcentagem simples para quantificação de variáveis e expostos em forma de gráficos e tabelas.

A pesquisa respeitou os aspectos éticos estabelecidos pela Resolução $\mathrm{N}^{\circ} 466 /$ 2012, do Conselho Nacional de Saúde (CNS), regulamentadoras de pesquisa envolvendo seres humanos. Os participantes assinaram o Termo de Consentimento Li-

\section{A amostra geral}

do estudo

contou com a

participação dos

28 colaboradores

da UAN,

representando

a totalidade dos

manipuladores

do local, sendo

$19(67,85 \%)$ do

sexo feminino e 9

$(32,14 \%)$ do sexo

masculino. vre e Esclarecido (TCLE) contendo todas as informações necessárias em linguagem clara, objetiva e de fácil entendimento. $\mathrm{O}$ presente estudo passou por avaliação do Comitê de Ética e Pesquisa do Hospital de Saúde Mental Professor Frota Pinto para liberação do termo de anuência e ao Comitê de Ética da Universidade de Fortaleza (PARECER N ${ }^{\circ}$ 24279719.7.0000.5038).

\section{RESULTADOS}

A amostra geral do estudo contou com a participação dos 28 colaboradores da UAN, representando a totalidade dos manipuladores do local, sendo 19 $(67,85 \%)$ do sexo feminino e $9(32,14 \%)$ do sexo masculino.

Por se tratar de uma unidade de alimentação hospitalar, as funções dos manipuladores são divididas em: copeiro $(n=8)$, cozinheiro $(n=6)$, auxiliares de cozinha $(n=10)$, estoquista $(n=1)$ e serviços gerais $(n=3)$.

Em relação ao nível de escolaridade dos colaboradores, metade da amostra $(n=14)$ possuía o ensino fundamental e o demais ensino médio $(\mathrm{n}=13)$. Apenas um colaborador possuía ensino técnico $(\mathrm{n}=1)$.

Em relação à presença de doenças crônicas $21,42 \%(n=6)$ dos colaboradores responderam já possuir diagnóstico confirmado de diabetes e/ou hipertensão. Sobre a prática regular de atividade física,

Quadro 1. Classificação do sobrepeso e obesidade segundo IMC, Circunferência da cintura e risco de doença associado. OMS, 2008.

\begin{tabular}{|c|c|c|c|c|}
\hline \multirow{4}{*}{ CLASSIFICAÇÃO } & \multirow{4}{*}{$\begin{array}{c}\text { IMC } \\
\text { (KG/M2) }\end{array}$} & \multirow{4}{*}{$\begin{array}{l}\text { CLASSE DA } \\
\text { OBESIDADE }\end{array}$} & \multicolumn{2}{|c|}{ RISCO PARA DIABETES TIPO II, HIPERTENSÃO E DOENÇA CARDIOVASCULAR } \\
\hline & & & CINTURA & CINTURA \\
\hline & & & $<102(\mathrm{M})$ & $>102(\mathrm{M})$ \\
\hline & & & $<88$ (F) & $>88$ (F) \\
\hline Baixo peso & 18.5 & & & \\
\hline Normal & $18.5-24.9$ & & & * \\
\hline Sobrepeso & 25.0-29.9 & & Aumentado & Alto \\
\hline \multirow{2}{*}{ Obesidade } & $30.0-34.9$ & I & Alto & Muito alto \\
\hline & 35.0-39.9 & ॥ & Muito alto & Muito alto \\
\hline Obesidade extrema & $>40$ & III & Extremamente alto & Extremamente alto \\
\hline
\end{tabular}


apenas $28,57 \%(n=8)$ afirmaram realizar pelo menos 3 vezes por semana com duração mínima de 30 minutos.

A avaliação do perfil antropométrico segundo o Índice de Massa Corporal (IMC) de acordo com os parâmetros da OMS (2008) está representada na tabela 1.

A tabela 2 apresenta a classificação do risco para surgimento de doenças crônicas como diabetes e hipertensão nos manipuladores de alimentos da unidade hospitalar de acordo com os critérios estabelecidos pela OMS (2008).

Quanto ao risco de desenvolvimento de doenças cardiovasculares através da aferição da circunferência da cintura, o maior percentual foi do sexo feminino que mostrou que 12 colaboradoras (42,85\%) apresentaram alto risco. Além disso, 4 colaboradores de ambos sexos apresentaram risco moderado.

\section{DISCUSSÃO}

O conhecimento ampliado sobre a saúde dos trabalhadores de uma forma geral, vem sendo aprimorado com o avanço da epidemiologia. Através dela, procura-se entender o processo saúde-

\section{Tabela 1 Classificação do IMC por sexo em manipuladores de alimentos de} uma unidade de nutrição hospitalar em Fortaleza-CE, 2020.

$$
\begin{aligned}
& \text { CLASSIFICAÇÃO POR SEXO } \\
& \text { HOMEM }
\end{aligned}
$$

$\begin{array}{ccccc} & \text { Baixo Peso } & 0 & 0 & 0 \\ \text { IMC } & \text { Eutrofia } & 03(10,71 \%) & 09(32,14 \%) & 12(42,85 \%) \\ & \text { Sobrepeso } & 02(7,14 \%) & 07(25 \%) & 09(32,14 \%) \\ & \text { Obesidade } & 04(14,28 \%) & 03(10,71 \%) & 07(25 \%)\end{array}$

* Os valores percentuais foram calculados levando em consideração o total de colaboradores, $n=28$.

Tabela 2 Classificação da Circunferência da Cintura (CC) e o risco de surgimento de doenças crônicas de acordo com os critérios da OMS (2008) em manipuladores de alimentos. Fortaleza-CE,2020.

\begin{tabular}{cccc} 
& \multicolumn{2}{c}{ TURNOS DE TRABALHO } & \multicolumn{1}{c}{ TOTAL } \\
& HOMENS & MULHERES & \\
Alto risco & $1(3,57 \%)$ & $12(42,85 \%)$ & $13(46,42 \%)$ \\
CC $\quad$ Risco Moderado & $4(14,28 \%)$ & $4(14,28 \%)$ & $8(28,57 \%)$ \\
Eutrófico & $4(14,28 \%)$ & $3(10,71 \%)$ & $7(25 \%)$ \\
* Os valores percentuais foram calculados levando em consideração o total de colaboradores, $\mathrm{n}=28$. &
\end{tabular}

-doença não apenas em cada indivíduo isoladamente (olhar clínico), mas também como esse processo se distribui entre os trabalhadores enquanto coletividade e classe social ${ }^{6,19}$

O excesso de peso, a obesidade e o sedentarismo são fatores de risco importantes para o surgimento de doenças crônicas como a diabetes, hipertensão e acidentes cardiovasculares. Dessa forma, a atenção dos profissionais de saúde em traçar estratégias que diminuam e previnam esses fatores na população brasileira são tratados como prioridades $8,16,17$

Dados da pesquisa Vigitel realizada pelo Ministério da Saúde no ano de 2016 e divulgados em 2017 apontam que a obesidade passou de $11,8 \%$ na população brasileira no ano de 2006 para 20,3\% em 2016. A mesma pesquisa apontou o alarmante dado que $55,4 \%$ da população está com excesso de peso ${ }^{10}$

A aferição do IMC e a medida da circunferência da cintura são estratégias simples utilizadas para caracterizar o perfil antropométrico da população que podem ser facilmente aplicadas em qualquer ambiente laboral. As informações provenientes dessas variáveis podem indicar a urgência de medidas que previnam agravos crônicos na população. ${ }^{5,6,10}$

A partir da aferição da circunferência da cintura é possível presumir os possíveis riscos de desenvolvimento de doenças cardiovasculares. Quanto maior a medida, maior a probabilidade dos exames bioquímicos do indivíduo apresentarem colesterol alto, diabetes e/ou hipertensão. ${ }^{7,8,10}$.

A pesquisa demonstrou que mais da metade da amostra composta por manipuladores de alimentos apresentou sobrepeso e/ou obesidade. Esse dado confirma a informação de Paiva e Cruz (2009) que sugerem que o aumento de peso é comum nessa classe de trabalhadores em consequência da natureza do trabalho, juntamente com a mudança de hábitos alimentares ${ }^{11}$

Um trabalho realizado em São José do Rio Preto- SP com 54 manipuladores de alimentos de uma UAN institucional em 2019, relatou que 59\% 


\section{artigo}

Brito, F.C.R.; Morira, M.R.; Ferreira da Silva, M.F.; Andrade, V.O.A.; Lima, W.O.O.

Perfil antropometrico e o risco de doenças cardiovasculares entre manipuladores de alimentos

$(\mathrm{n}=32)$ da amostra estava com sobrepeso e/ou algum grau de obesidade, segundo o critério da $\mathrm{OMS}^{12}$. Assim como na presente pesquisa, a maioria dos participantes com sobrepeso eram do sexo feminino.

A educação em saúde constante é uma das estratégias utilizadas pelos gestores de saúde para prevenir agravos à saúde do trabalhador. Os manipuladores de alimentos, devem ter acesso constante às ações educativas que estimulem o hábito da atividade física e a mudança de hábitos alimentares. ${ }^{13,14,15}$

\section{CONCLUSÃO}

O perfil antropométrico dos manipuladores de alimentos da unidade estudada demonstrou risco alto para o surgimento de agravos relacionados ao excesso de peso.

Sugerimos o aprimoramento de pesquisas que caracterizem o perfil antropométrico, incluindo fatores relacionados aos hábitos alimentares, consumo de alimentos de baixa qualidade nutricional, ingestão de bebida alcoólica e tabagismo; itens não abordados nesta pesquisa e que podem ampliar na caracterização do perfil de saúde desses trabalhadores.

Pesquisas como esta podem auxiliar na implantação de políticas públicas e normativas que incentivem e fortaleçam a educação em saúde, melhorando, assim, a qualidade de vida dos manipuladores de alimentos.

\section{REFERÊNCIAS}

1.Estevam E et al. Caracterização do perfil nutricional e dos aspectos ergonômicos relacionados ao trabalho de colaboradores de uma unidade de alimentação e nutrição. Revista Científica da Faminas, 2013; 9(2):55-68.

2.Strasbourg VJ. et al. Avaliação de condições ergonômicas em trabalhos realizados em restaurantes universitários. Revista Eletrônica em Gestão, Educação e Tecnologia Ambiental.2015; 19(3): 900-910.

3.Símon MJ et al. Avaliação nutricional dos profissionais do serviço de nutrição e dietética de um hospital terciário de Porto Alegre. Card. Saúde Coletiva. 2014;22(1):69-74

4.Paixão MPCP.et al.. Obesidade como fator de risco para acidentes no trabalho. Revista Saúde e Pesquisa.2009; 2(3): 379-386, 2009.

5. World Health Organization. Obesity: preventing and managing the global epidemic. Report of a World Health Organization Consultation. Geneva: World Health Organization, 2000. 253p.

6. Mendes R e Dias EC.Saúde do Trabalhador In:ROUQUAYROL, M.Z. Introdução a epidemiologia. 9 ed. Rio de Janeiro: Guanabara Koogan, 2006.

7. Associação Brasileira para o Estudo da Obesidade e da Síndrome Metabólica. Diretrizes brasileiras de obesidade. ABESO - Associação Brasileira para o Estudo da Obesidade e da Síndrome Metabólica. 2016 - São Paulo. $4^{\circ}$ ed.

8.Kazapi IAM et al. Estado nutricional dos comensais e adequação da refeição servida no restaurante universitário da Universidade Federal de Santa Catarina, com proposta de cardápios de baixo custo. 2004. Revista Nutrição em Pauta 67(3):34-40.

9. Mello AG et al. Conhecimentos dos manipuladores de alimentos sobre boas práticas nos restaurantes públicos populares do Estado do Rio de Janeiro.2010. BrazilianJournal of Food Technology. (3):1, p. 60-68.

10. Brasil. Ministério da Saúde. Vigitel Brasil 2016. Saúde Suplementar: vigilância de fatores de risco e proteção para doenças crônicas por inquérito telefônico [recurso eletrôni- co].2017 Ministério da Saúde, Agência Nacional de Saúde Suplementar.

11. Paiva AC e Cruz AA. Estado nutricional e aspectos Ergonômicos de trabalhadores de Unidade de alimentação e Nutrição.2009. Revista Mineira de Ciências da Saúde; 1(1): 1-9.

12. Vieira ACM e Costa T. Perfil nutricional de manipuladores de alimentos. Revista Científica Unilagos. 2019 1(1): 2-13.

13. Silva PC, zaffarl D. Prevalência de excesso de peso e associação com outras variáveis em indivíduos adultos atendidos em unidade básica de saúde. Sci Med. 2009;19(1):17-26.

14. Souza L J. de, GNC, Chalita F EB., Reis A FF., Bastos DA., Souto FJ T.D. et al .Prevalência de Obesidade e fatores de risco cardiovascular em Campos, Rio de Janeiro. ArqBrasEndocrinoMetab. 2003; 47(6):669-76.

15.Cercato C, MANCINI MC, ARGUELLO AMC, PASSOS VQ, VILLARES SMF, HALPERN A. Systemichypertension, diabetes mellitus, anddyslipidemia in relationtobodymass index: evaluationof a Brazilianpopulation.RevHospClin. 2004;59(3):113-8.

16. Baena C. P., Muccillo-Baisch A.L., Almeida T.L., De La Rocha C, Franco O.S, Olmedo D. et al et al. Impacto de um programa piloto de promoção da saúde para trabalhadores marítimos de rebocadores. RevBras Saúde Ocup. 2011;36(124):288-96.

17. Moraes KD, Araujo AP, Santos AF, Barbosa JM, Martins $\mathrm{ML}$. Correlação entre o índice de massa corporal e indicadores antropométricos de risco cardiovascular em mulheres. RevPesq Saúde. 2015;16(3):175-81.

18. Silva ST. Fatores de risco cardiovasculares em usuários do hiperdia de uma unidade básica de saúde do município de Criciúma/SC. [Monografia]. Universidade do Extremo Sul Catarinense, Florianópolis; 2012.

19. Cristóvão MF, Sato APS, Fujimorl E. Excesso de peso e obesidade abdominal em mulheres atendidas em unidade da estratégia de saúde da família.RevEscEnferm. 2011;45(2):1667-72. 